\title{
JACOBSON RADICALS OF NEST ALGEBRAS IN FACTORS
}

\author{
XINGDE DAI
}

(Communicated by Palle E. T. Jorgensen)

\begin{abstract}
Definition. Let $\beta$ be a nest in a separably acting type $\mathrm{II}_{\infty}$ factor $\mathscr{M}$. An element $P \in \beta \backslash\{0, I\}$ is said to be a singular point of $\beta$ if it satisfies either of the following conditions:

(1) There is a strictly increasing sequence $\left\{Q_{n}\right\} \subseteq \beta, \lim _{n \rightarrow \infty} Q_{n}=P$, and $P-Q_{n}$ is infinite for each $n \in \mathrm{N}$. Also, there is a projection $Q \in \beta$ such that $Q>P$ and $Q-P$ is finite.

(2) There is a strictly decreasing sequence $\left\{Q_{n}\right\} \subseteq \beta, \lim _{n \rightarrow \infty} Q_{n}=P$, and $Q_{n}-P$ is infinite for each $n \in \mathrm{N}$. Also, there is a projection $Q \in \beta$ such that $Q<P$ and $P-Q$ is finite.

Main Theorem. Let $\beta$ be a nest in a separably acting factor $\mathscr{M}$.

(1) If $\mathscr{M}$ is of type $\mathrm{II}_{\infty}$, then a necessary and sufficient condition for the Jacobson radical $\mathscr{R}_{\beta}$ of alg $\beta$ to be a norm-closed singly generated ideal of $\operatorname{alg} \beta$ is that the nest $\beta$ is countable and it does not contain a singular point.

(2) If $\mathscr{K}$ is of type $\mathrm{II}_{1}$ or type $\mathrm{III}$, then a necessary and sufficient condition for the Jacobson radical $\mathscr{R}_{\beta}$ of $\operatorname{alg} \beta$ to be a norm-closed singly generated ideal of $\operatorname{alg} \beta$ is that the nest $\beta$ is countable.
\end{abstract}

(3) In (1) and (2) the single generation is equivalent to countable generation.

\section{INTRODUCTION}

The nest algebra in a von Neumann algebra was introduced by Gilfeather and Larson [5]. They extend the Ringrose Criterion [7] of the membership for the Jacobson radical of a nest algebra into the von Neumann algebra setting.

In the $\mathscr{B}(H)$ case Orr [6] characterized necessary and sufficient conditions for a general nest to have the property that the Jacobson radical is norm-closed singly generated. In this paper we will establish conditions for the Jacobson radicals $\mathscr{R}_{\beta}$ of alg $\beta$ in separably acting type II and type III factors.

\section{Preliminaries}

Let $\mathscr{M}$ be a factor acting on separable Hilbert space $\mathscr{H}$. By a nest in we mean a totally ordered family of (selfadjoint) projections containing $\{0, I\}$

Received by the editors April 9, 1992; presented at GPOTS, Iowa, 1992.

1991 Mathematics Subject Classification. Primary 47B99.

This work was supported in part by funds from the Foundation of UNCC and State of North Carolina. 
which is closed in the strong operator topology. A nest $\beta$ is countable if it is a countable set. The nest algebra related to a nest $\beta$ in a factor $\mathscr{M}$ is the set

$$
\operatorname{alg} \beta=\{T \in \mathscr{M}: T P=P T P \text { for each } P \in \beta\} \text {. }
$$

Definition 1. Let $\beta$ be a nest in a separably acting type $\mathrm{II}_{\infty}$ factor $\mathscr{M}$. An element $P \in \beta \backslash\{0, I\}$ is said to be a singular point of $\beta$ if it satisfies either of the following conditions:

(1) There is a strictly increasing sequence $\left\{Q_{n}\right\} \subseteq \beta, \lim _{n \rightarrow \infty} Q_{n}=P$, and $P-Q_{n}$ is infinite for each $n \in \mathbb{N}$. Also, there is a projection $Q \in \beta$ such that $Q>P$ and $Q-P$ is finite.

(2) There is a strictly decreasing sequence $\left\{Q_{n}\right\} \subseteq \beta, \lim _{n \rightarrow \infty} Q_{n}=P$, and $Q_{n}-P$ is infinite for each $n \in \mathbb{N}$. Also, there is a projection $Q \in \beta$ such that $Q<P$ and $P-Q$ is finite.

The following theorem is our main result in this paper.

Theorem 1. Let $\beta$ be a nest in a separably acting factor $\mathscr{M}$.

(1) If $\mathscr{M}$ is of type $\mathrm{II}_{\infty}$, then a necessary and sufficient condition for the Jacobson radical $\mathscr{R}_{\beta}$ of alg $\beta$ to be a norm-closed singly generated ideal of $\operatorname{alg} \beta$ is that the nest $\beta$ is countable and it does not contain a singular point.

(2) If $\mathscr{M}$ is of type $\mathrm{II}_{1}$ or type III, then a necessary and sufficient condition for the Jacobson radical $\mathscr{R}_{\beta}$ of alg $\beta$ to be a norm-closed singly generated ideal of $\operatorname{alg} \beta$ is that the nest $\beta$ is countable.

(3) In (1) and (2) single generation is equivalent to countable generation.

Let $\mathscr{M}$ be a factor in $\mathscr{B}(H)$ for a separable Hilbert space $\mathscr{H}$ and $\beta$ be a nest in $\mathscr{M}$. We can write $\beta=\left\{N_{\lambda}: \lambda \in \Lambda\right\}$ for some closed set $\Lambda \subseteq[0,1]$ which contains 0 and 1 . A net $\left\{N_{\lambda_{t}}\right\}$ in $\beta$ converges to $N_{t_{0}} \in \beta$ in the strong operator topology if and only if $\lambda_{t}$ converges to $t_{0}$ in the regular topology on $[0,1]$. A projection $E=M-N, M, N \in \beta, M>N$, is called a $\beta$ interval. The projections $M, N$ are called the upper and lower end points of $E$, respectively.

The Jacobson radical of an arbitrary algebra is defined to be the intersection of the kernels of all strictly transitive representations of the algebra. The radical of a Banach algebra is a closed 2-sided topologically nil-ideal which contains every topologically nil left or right ideal in the algebra. If $\mathscr{A}$ is a unital Banach algebra and $\mathscr{R}$ is its radical, then

$$
\begin{aligned}
\mathscr{R} & =\{B \in \mathscr{A}: A B \text { is quasinilpotent, } A \in \mathscr{A}\} \\
& =\{B \in \mathscr{A}: B A \text { is quasinilpotent, } A \in \mathscr{A}\} .
\end{aligned}
$$

Also, if $\sigma(A)$ denotes the spectrum of $A$ in $\mathscr{A}$, then $B \in \mathscr{R}$ if and only if $\sigma(A+B)=\sigma(A)$ for all $A \in \mathscr{A}$.

In [5], Gilfeather and Larson generalized the Ringrose Criterion [7] to the radical $\mathscr{R}_{\beta}$ of alg $\beta$ into the von Neumann algebra setting. They proved:

Theorem 2 (Gilfeather, Larson, Ringrose [5,7]). If $A \in \operatorname{alg} \beta$, then $A \in \mathscr{R}_{\beta}$ if and only if for each $\varepsilon>0$ there exists a finite set $\left\{E_{i}\right\}$ of mutually orthogonal $\beta$-intervals with $\sum E_{i}=I$ such that $\left\|E_{i} A E_{i}\right\|<\varepsilon$ for all $i$. 


\section{SUFFICIENCY}

Let $\beta$ be a nest in a factor $\mathscr{M}$. We will say that an element $N$ in a nest $\beta$ is a left limit point of $\beta$ if there is a strictly increasing sequence $\left\{N_{n}\right\}$ in $\beta$ such that $N$ is the sot(strong operator)-limit of $\left\{N_{n}\right\}$.

We will use the following notation:

$$
\begin{aligned}
& E_{\lambda}(\beta)=\bigvee\{N \in \beta: N \text { is finite }\}, \\
& E_{\rho}(\beta)=\bigvee\left\{N^{\perp}: N \in \beta \text { and } N^{\perp} \text { is finite }\right\} .
\end{aligned}
$$

Definition 2. Let $\beta$ be a nest in a type $\mathrm{II}_{\infty}$ factor $\mathscr{M}$.

(1) The nest is said to be of order type $I_{1}$ if it is an infinite set, and either $\beta$ or its dual $\beta^{\perp}$ satisfies the following conditions:

(a) Both $E_{\lambda}$ and $E_{\rho}^{\perp}$ of the nest are left limit points of the nest.

(b) The projection $E_{\lambda}$ is infinite and the projection $E_{\rho}$ is finite.

(2) The nest is said to be of order type $I_{2}$ if $\beta$ or its dual nest $\beta^{\perp}$ satisfies the following condition:

The identity $I$ is a limit point of the nest. If $Q$ is in the nest, $Q<I$, $Q^{\perp}$ is infinite. There exists a finite nonzero projection $P$ in the nest. $\mathrm{I}_{2}$.

(3) $\beta$ is said to be of order type $I$ if it is of order type $I_{1}$ or of order type

(4) $\beta$ is said to be of order type II if it is not of order type I.

The main results in [2] are concerned with the extension of the results in [1] into the von Neumann algebra setting. The author proved the following

Theorem 3 [1]. Let $\beta$ be a nest in a separably acting factor $\mathscr{M}$.

(1) Assume that $\mathscr{M}$ is of type $\mathrm{II}_{\infty}$. Then the factor $\mathscr{M}$ is a norm-principal (or norm-closed singly generated) bimodule of $\operatorname{alg} \beta$ if and only if the nest $\beta$ is of order type II.

(2) Assume that $\mathscr{M}$ is of type II $_{1}$ or type III. Then the factor $\mathscr{M}$ is always a norm-principal bimodule of alg $\beta$.

Let $\beta$ be a nest in $\mathscr{M}$ and let $P \in \beta \backslash\{0, I\}$. We define a set of projections $\beta_{P}$ in $\mathscr{M}$ by

$$
\beta_{P}=\left\{P^{\perp}+N: N \leq P, N \in \beta\right\} \cup\{N-P: N>P, N \in \beta\} \cup\{0, I\} .
$$

It is clear $\beta_{P}$ is also a nest.

The nest $\beta_{P}$ has some properties which are important to the purpose in this paper.

Lemma 1. Let $\beta$ be a nest in $\mathscr{M}$ and let $P \in \beta \backslash\{0, I\}$. Then

(1) $\left(\beta_{P}\right)_{P^{\perp}}=\beta$;

(2) $P(\operatorname{alg} \beta) P=P\left(\operatorname{alg} \beta_{P}\right) P$;

(3) $P^{\perp}(\operatorname{alg} \beta) P^{\perp}=P^{\perp}\left(\operatorname{alg} \beta_{P}\right) P^{\perp}$.

Proof. Statement (1) is obvious.

Let $T \in P(\operatorname{alg} \beta) P$ and $N \in \beta_{P}$. If $N \geq P^{\perp}$, then there is $M \in \beta, M<$ $P$, and $N=P^{\perp}+M$. So $T N=P T P N=P T P\left(P^{\perp}+M\right)=P T M N=$ 
$P M T M N=N T N$. If $N<P^{\perp}$, then $N=M-P$ for some $M>P$ and $M \in \beta$. So $T N=P T P N=0=N T N$. So we have $T \in \operatorname{alg} \beta_{P}$. Hence

$$
P(\operatorname{alg} \beta) P \subseteq P\left(\operatorname{alg} \beta_{P}\right) P .
$$

By statement (1) and (1) we have

$$
P^{\perp}\left(\operatorname{alg} \beta_{P}\right) P^{\perp} \subseteq P^{\perp}(\operatorname{alg} \beta) P^{\perp} .
$$

Let $T \in P\left(\operatorname{alg} \beta_{P}\right) P$ and $M \in \beta$. If $M<P$, then $P^{\perp}+M \in \beta_{P}$. So $T M=$ $P T P M=P T\left(P^{\perp}+M\right) P=P\left(P^{\perp}+M\right) T\left(P^{\perp}+M\right) P=M T M$. If $M \geq P$, then $M P=P M=P$, so $M T M=T=T M$. So we have $T \in \operatorname{alg} \beta$, and

$$
P(\operatorname{alg} \beta) P \supseteq P\left(\operatorname{alg} \beta_{P}\right) P \text {. }
$$

By statement (1) and (3) we have

$$
P^{\perp}\left(\operatorname{alg} \beta_{P}\right) P^{\perp} \supseteq P^{\perp}(\operatorname{alg} \beta) P^{\perp} .
$$

Lemma 1 is proven.

Lemma 2. Let $\beta$ be a nest in a type $\mathrm{II}_{\infty}$ factor $\mathscr{M}$ and let $P \in \beta \backslash\{0, I\}$ which is not a singular point.

(1) If there is a projection $Q \in \beta$ such that $Q-P$ is finite and, for each projection $R<P, R \in \beta$ and the projection $P-R$ is infinite, then $P$ has an immediate predecessor $P_{-}$in $\beta$ and $P-P_{-}$is an infinite atom of $\beta$.

(2) If there is a projection $Q \in \beta$ such that $P-Q$ is finite and, for each projection $R>P, R \in \beta$ and the projection $R-P$ is infinite, then $P$ has an immediate successor $P_{+}$in $\beta$ and $P_{+}-P$ is an infinite atom of $\beta$.

Proof. By the definition of the singular point, it is clear.

Lemma 3. Let $\beta$ be a nest in a type $\mathrm{II}_{\infty}$ factor $\mathscr{M}$ and let $P \in \beta \backslash\{0, I\}$ with both $P$ and $P^{\perp}$ infinite. Assume that $\beta$ has no singular point. Then $\beta_{P}$ is an order type II nest in $\mathscr{M}$.

Proof. Define

$$
\begin{aligned}
& \lambda_{P}^{-}=\bigwedge\{Q \in \beta: P-Q \text { is finite }\} \\
& \lambda_{P}^{+}=\bigvee\{Q \in \beta: Q-P \text { is finite }\}
\end{aligned}
$$

For the projections just defined, we have the following cases.

For $\lambda_{P}^{-}$, we have:

(1) $\lambda_{P}^{-}=0$ and $P-\lambda_{P}^{-}$is infinite.

(2) $\lambda_{P}^{-}>0$ and $P-\lambda_{P}^{-}$is finite.

(3) $\lambda_{P}^{-}>0$ and $P-\lambda_{P}^{-}$is infinite.

For $\lambda_{P}^{+}$, we have:

(1) $\lambda_{P}^{+}=I$ and $\lambda_{P}^{+}-P$ is infinite.

(2) $\lambda_{P}^{+}<I$ and $\lambda_{P}^{+}-P$ is finite.

(3) $\lambda_{P}^{+}<I$ and $\lambda_{P}^{+}-P$ is infinite.

It is clear that the case (1)-(2) is the dual of $(2)-(1)$; the case (1)-(3) is the dual of (3)-(1); and the case (2)-(3) is the dual of (3)-(2). Notice that a nest is of order type II if and only if its dual has order type II and a nest has no 
singular point if and only if its dual has no singular point. It suffices to discuss the following six cases: (1)-(1), (1)-(2), (1)-(3), (2)-(2), (2)-(3), and (3)-(3).

Case (1)-(1). It is clear that $\beta_{P}$ is of order type II.

Case (1)-(2). Since $\lambda_{P}^{+}-P$ is finite and $P^{\perp}$ is infinite, by Lemma $2,\left(\lambda_{P}^{+}\right)_{+}-\lambda_{P}^{+}$ must be an infinite atom. So, $\beta_{P}$ is of order type II.

Case (1)-(3). It is clear.

Case (2)-(2). In this case $\lambda_{P}^{+}-\lambda_{P}^{-}$is finite and $P$ and $P^{\perp}$ are infinite. So by Lemma 2 again $\left(\lambda_{P}^{+}\right)_{+}-\lambda_{P}^{+}$and $\lambda_{F}^{-}-\left(\lambda_{P}^{-}\right)_{-}$are infinite atoms. So, $\beta_{P}$ is of order type II.

Case (2)-(3). Similar to Case (2)-(2).

Case (3)-(3). It is clear.

Lemma 3 is proven.

Let $\mathscr{I}_{c}$ be the (only) norm-closed nontrivial ideal in a type $\mathrm{II}_{\infty}$ factor $\mathscr{M}$. In [2] the author proved

Lemma 4 [2]. Let $\beta$ be a nest in a type $\mathrm{II}_{\infty}$ factor $\mathscr{M}$. A necessary and sufficient condition for $\mathscr{I}_{c}$ to be a norm-closed singly generated bimodule of alg $\beta$ is that the nest does not satisfy either one of the following conditions:

- The 0 projection is a limit point of the nest and any nonzero projection in $\beta$ is infinite.

- The identity $I$ is a limit point of the nest and, for any projection $Q<$ $I, Q \in \beta, Q^{\perp}$ is infinite.

Proposition 1. Let $\beta$ be a nest in a type $\mathrm{II}_{\infty}$ factor $\mathscr{M}$ and let $P \in \beta \backslash\{0, I\}$. Assume that $\beta$ has no singular point. Then there exists an operator $G_{P} \in$ $P(\operatorname{alg} \beta) P^{\perp} \subseteq \mathscr{R}_{\beta}$ such that

$$
P \mathscr{M} P^{\perp}=P(\operatorname{alg} \beta) P^{\perp}=P\left(\mathscr{R}_{\beta}\right) P^{\perp}=\left[(\operatorname{alg} \beta) G_{P}(\operatorname{alg} \beta)\right] .
$$

Proof. (1) Assume both $P$ and $P^{\perp}$ are infinite. By Lemma 3 and Theorem 3, there is an operator $R \in \mathscr{M}$ such that $\mathscr{M}=\left[\left(\operatorname{alg} \beta_{P}\right) R\left(\operatorname{alg} \beta_{P}\right)\right]$.

Recall that $P^{\perp} \in \beta_{P}$; we have

$$
\begin{aligned}
P \mathscr{M} P^{\perp} & =P(\operatorname{alg} \beta) P^{\perp}=P\left[\left(\operatorname{alg} \beta_{P}\right) R\left(\operatorname{alg} \beta_{P}\right)\right] P^{\perp} \\
& =\left[P\left(\operatorname{alg} \beta_{P}\right) P R P^{\perp}\left(\operatorname{alg} \beta_{P}\right) P^{\perp}\right] \\
& =\left[P\left(\operatorname{alg} \beta_{P}\right) P\left(P R P^{\perp}\right) P^{\perp}\left(\operatorname{alg} \beta_{P}\right) P^{\perp}\right] .
\end{aligned}
$$

Let $G_{P}=P R P^{\perp}$. By Lemma 1 we have

$$
\begin{aligned}
P \mathscr{M} P^{\perp} & =P(\operatorname{alg} \beta) P^{\perp}=P\left(\mathscr{R}_{\beta}\right) P^{\perp} \\
& =\left[P(\operatorname{alg} \beta) P G_{P} P^{\perp}(\operatorname{alg} \beta) P^{\perp}\right]=\left[(\operatorname{alg} \beta) G_{P}(\operatorname{alg} \beta)\right] .
\end{aligned}
$$

(2) Assume that $P$ is finite. (The case when $P^{\perp}$ is finite is a dual case to this. We omit it.) Then $P \mathscr{M} P^{\perp}=P \mathscr{J}_{c} P^{\perp}$. Since $\beta$ has no singular point, we have only two possible cases:

(a) There is a projection $Q \in \beta, P<Q$, and $Q-P$ is finite. 
(b) For each projection $Q>P, Q \in \beta$, the projection $Q-P$ is infinite. Then by Lemma $2 P$ is a left end point of an infinite atom of $\beta$.

It is clear that in either case the nest $\beta_{P}$ satisfies the condition in Lemma 4. By Lemma 4 there exists an operator $R \in \mathscr{M}$ such that

$$
\mathscr{I}_{c}=\left[\left(\operatorname{alg} \beta_{P}\right) R\left(\operatorname{alg} \beta_{P}\right)\right] \text {. }
$$

Therefore,

$$
P \mathscr{M} P^{\perp}=P \mathscr{I}_{c} P^{\perp}=P\left[\left(\operatorname{alg} \beta_{P}\right) R\left(\operatorname{alg} \beta_{P}\right)\right] P^{\perp} .
$$

Now the same technique we used in part (1) applies. Proposition 1 is proven.

Proposition 2. Let $\beta$ be a countable nest in a separably acting factor $\mathscr{M}$. If for each $P \in \beta \backslash\{0, I\}$ we have $P(\operatorname{alg} \beta) P^{\perp}=\left[(\operatorname{alg} \beta) G_{P}(\operatorname{alg} \beta)\right]$ for some operator $G_{P} \in \operatorname{alg} \beta$, then the Jacobson radical $\mathscr{R}_{\beta}$ is a norm-principal ideal of alg $\beta$.

Proof. The proof is the same as that of Proposition 4 in [1]. The reader is referred to the proof in [1].

By Theorem 3 and Propositions 1 and 2, the sufficient part of Theorem 1 is proven.

\section{NECESSITY}

In this section we will first show that if the Jacobson radical is a normprincipal ideal of $\operatorname{alg} \beta$, then the nest must be countable.

Proposition 3. Let $\beta$ be a nest in a separably acting factor $\mathscr{M}$. If $\mathscr{R}_{\beta}$ is a normclosed countably generated ideal of alg $\beta$, then the nest $\beta$ must be countable.

Proof. Let $\beta=\left\{N_{\lambda}: \lambda \in \Lambda \subset[0,1]\right\}$. Let $\phi:(\operatorname{alg} \beta) \times[0,1] \rightarrow \mathbb{R}^{+}$be Erdos's diagonal function of $\beta$, defined as follows. Given $A \in \operatorname{alg} \beta$ and $t \in(0,1)$,

$$
\begin{aligned}
& \phi(A, t)=\inf \left\{\left\|N\left[\lambda_{1}, \lambda_{2}\right] A N\left[\lambda_{1}, \lambda_{2}\right]\right\|: \lambda_{1}, \lambda_{2} \in \Lambda, \lambda_{1}<t<\lambda_{2}\right\}, \\
& \phi(A, 0)=\inf \left\{\left\|N_{\lambda} A N_{\lambda}\right\|: \lambda \in \Lambda, 0<\lambda\right\}, \\
& \phi(A, 1)=\inf \left\{\left\|\left(I_{\mathscr{M}}-N_{\lambda}\right) A\left(I_{\mathscr{M}}-N_{\lambda}\right)\right\|: \lambda \in \Lambda, \lambda<1\right\} .
\end{aligned}
$$

Assume that $\mathscr{G}=\left\{G_{n}\right\}$ is a countable subset in $\mathscr{R}_{\beta}$ and let $G \in \mathscr{G}$. Employing the diagonal function $\phi$ and the same method in the proof of Proposition 6 in [1], we prove that $S_{G}=\{\lambda \in \Lambda: \phi(G, \lambda) \neq 0\}$ is a countable set. So $S_{\mathscr{G}}=\bigcup S_{G_{n}}$ is a countable subset of $\Lambda$. Let $\mathscr{I}_{\mathscr{G}}$ denote the norm-closed ideal generated by $\mathscr{G}$ and let $A \in \mathscr{I}_{\mathscr{G}}$. Applying the same method in the proof of Proposition 6 in [1] or in [6], we prove that $S_{A} \subseteq S_{\mathscr{G}}$.

Assume $\beta$ is an uncountable nest. We will show that $\mathscr{I}_{\mathscr{G}}$ is a proper subset of $\mathscr{R}_{\beta}$. Since $\Lambda$ is an uncountable compact subset of $\Lambda$, there exists a point $t_{0} \in \Lambda \backslash\left(S_{\mathscr{G}} \cup\{0,1\}\right)$ and $t_{0}$ is a two-sided limit point of $\Lambda$. Let $\left\{\alpha_{n}\right\}_{n=1}^{\infty}$ be a strictly increasing sequence in $\Lambda$ converging to $t_{0}$ and let $\left\{\beta_{n}\right\}_{n=1}^{\infty}$ be a strictly decreasing sequence in $\Lambda$ converging to $t_{0}$.

Denote $E_{n}=N_{\alpha_{n+1}}-N_{\alpha_{n}}$ and $F_{n}=N_{\beta_{n}}-N_{\beta_{n+1}}$. Then for each $n \in \mathbb{N}$ there exist non-0 projections $E_{n}^{\prime} \leq E_{n}$ and $F_{n}^{\prime} \leq F_{n}$ such that $E_{n}^{\prime} \sim F_{n}^{\prime}$ for each $n \in \mathbb{N}$. Let $Q_{n}$ be a partial isometry with initial projection $F_{n}^{\prime}$ and final projection $E_{n}^{\prime}$. Define an operator $R_{0}=\sum_{n=1}^{\infty} Q_{n}$. The operator $R_{0}$ is in $\mathscr{R}_{\beta}$ and $t_{0} \in S_{R_{0}}$, so $t_{0} \in S_{\mathscr{G}}$. This contradicts the selection of $t_{0}$. Proposition 3 is proven. 
Next we will prove that if a nest $\beta$ in a type $\mathrm{II}_{\infty}$ factor has a singular point, then the radical $\mathscr{R}_{\beta}$ cannot be even countably generated.

Let $F$ be a fixed nonzero finite projection in the type $\mathrm{II}_{\infty}$ factor $\mathscr{M}$. Then there exists a faithful normal semifinite tracial weight $\varrho$ on $\mathscr{M}$ such that $\varrho(F)=1$ (cf. [4, Proposition 8.5.5]). For an operator $T \in \mathscr{M}$, if its range projection $R_{T}$ is finite, we define a range measure of $T$ by $\mu(T)=\varrho\left(R_{T}\right)$. If the range projection is infinite, we define $\mu(T)=\infty$. In [2] we proved the following

Lemma 5 [2]. Let $T$ and $S$ be finite range operators. Then:

(1) $\mu(T S) \leq \min \{\mu(S), \mu(T)\}$.

(2) $\mu(T+S) \leq \mu(S)+\mu(T)$.

(3) If $Q$ is a partial projection and $\mu(Q)>\mu(T)$, then $\|Q-T\| \geq 1$.

(4) If $\left\{N_{n}\right\}$ is a sequence of projections such that $N_{n} \rightarrow 0$ in the strong operator topology and $T \in \mathscr{M}$ has finite range projection $R_{T}$, then $\lim \mu\left(N_{n} T\right)=0$.

The following theorem together with Proposition 3 completes the proof of the necessary part of Theorem 1. Theorem 4 also provides a crucial step for the proof of Theorem 1(3).

Theorem 4. Let $\beta$ be a nest in a separably acting type $\mathrm{II}_{\infty}$ factor $\mathscr{M}$. Assume $\beta$ has a singular point and $\mathscr{G}$ be a sequence of operators in $\mathscr{R}_{\beta}$. Then $[(\operatorname{alg} \beta) \mathscr{G}(\operatorname{alg} \beta)]$ is a proper subset of $\mathscr{M}$.

Proof. (1) Let $\mathscr{G}=\left\{G_{n}\right\}$ be an arbitrary given sequence of operators in $\mathscr{R}_{\beta}$. Assume that $P$ is a singular point of $\beta$. We can assume that $\beta$ and $P$ satisfy Definition 1(1). (The other case is the dual to this.)

Assume that $\mathscr{R}_{\beta}=[(\operatorname{alg} \beta) \mathscr{G}(\operatorname{alg} \beta)]$. According to the assumption we have

$$
\begin{aligned}
(Q & \left.-P_{1}\right)\left(\mathscr{R}_{\beta}\right)\left(Q-P_{1}\right) \\
& =\left[\left(\left(Q-P_{1}\right)(\operatorname{alg} \beta)\left(Q-P_{1}\right)\right)\left(\left(Q-P_{1}\right) \mathscr{G}\left(Q-P_{1}\right)\right)\left(\left(Q-P_{1}\right)(\operatorname{alg} \beta)\left(Q-P_{1}\right)\right)\right] \\
& =\left[\left(\operatorname{alg}\left(\left(Q-P_{1}\right) \beta\right)\right)\left(\left(Q-P_{1}\right) \mathscr{G}\left(Q-P_{1}\right)\right)\left(\operatorname{alg}\left(\left(Q-P_{1}\right) \beta\right)\right)\right],
\end{aligned}
$$

where $\operatorname{alg}\left(\left(Q-P_{1}\right) \beta\right)$ is the nest algebra in the (type $\left.\mathrm{II}_{\infty}\right)$ factor $\left(Q-P_{1}\right) \times$ $\mathscr{M}\left(Q-P_{1}\right)$ related to the nest $\left(Q-P_{1}\right) \beta$. It is clear that $\left(Q-P_{1}\right)\left(\mathscr{R}_{\beta}\right)\left(Q-P_{1}\right)$ is the Jacobson radical of $\operatorname{alg}\left(\left(Q-P_{1}\right) \beta\right)$. So our assumption implies that the countable set $\left(Q-P_{1}\right) \mathscr{G}\left(Q-P_{1}\right)$ is a generating set for the radical $\mathscr{R}_{\left(Q-P_{1}\right) \beta}$. It is also clear that the projection $\left(Q-P_{1}\right) P$ is a singular point in the nest $\left(Q-P_{1}\right) \beta$. Therefore, we can simply assume that $P_{1}=0$ and $Q=I$. We assume this is the case. Under this new assumption $P^{\perp}$ is finite and $P$ is infinite, and

$$
\begin{aligned}
P \mathscr{M} P^{\perp} & =P(\operatorname{alg} \beta) P^{\perp}=P \mathscr{I}_{c} P^{\perp}=P \mathscr{R}_{\beta} P^{\perp} \\
& =P[(\operatorname{alg} \beta) \mathscr{G}(\operatorname{alg} \beta)] P^{\perp}=\mathscr{J}_{1}+\mathscr{I}_{2}+\mathscr{I}_{3},
\end{aligned}
$$

where

$$
\begin{aligned}
\mathscr{I}_{1} & =\left[(P \operatorname{alg} \beta P)(P \mathscr{G} P)\left(P \operatorname{alg} \beta P^{\perp}\right)\right], \\
\mathscr{I}_{2} & =\left[(P \text { alg } \beta P)\left(P \mathscr{G} P^{\perp}\right)\left(P^{\perp} \text { alg } \beta P^{\perp}\right)\right], \\
\mathscr{I}_{3} & =\left[\left(P \operatorname{alg} \beta P^{\perp}\right)\left(P^{\perp} \mathscr{G} P^{\perp}\right)\left(P^{\perp} \operatorname{alg} \beta P^{\perp}\right)\right] .
\end{aligned}
$$


(2) Now we construct an operator (a partial isometry) $T_{0} \in P \mathscr{M} P^{\perp}$ which is not in $P[(\operatorname{alg} \beta) \mathscr{G}(\operatorname{alg} \beta)] P^{\perp}$. This will complete the proof. We will use the techniques we developed in [2]. We have two cases.

Case 1. There is a strictly decreasing sequence of projections $\left\{Q_{n}\right\} \subset \beta$ such that $s-\lim _{n \rightarrow \infty} Q_{n}=P$. Let $\varrho$ be the faithful normal semifinite tracial weight on $\mathscr{M}$ such that $\varrho\left(P^{\perp}\right)=1$, and let $\mu$ be the related range measure. Then $\mu\left(P^{\perp}\right)=1$. We can assume that the sequence $\left\{P_{n}\right\}$ possesses the property $\mu\left(P_{n+1}-P_{n}\right) \geq 1$. Denote $\alpha_{n}=\mu\left(Q_{n}-Q_{n+1}\right)$, where $Q_{1}=I$. It is a routine exercise to verify that $\sum_{j=1}^{\infty} \alpha_{j}=1$. Notice that $\mu\left(P G_{n} P^{\perp} Q_{k}\right) \leq 1$ for $n, k \in \mathbb{N}$ and $\lim P_{n}=P$. For fixed $n, k \in \mathbb{N}$ and $\varepsilon>0$, by Lemma 5 there is an $n_{k} \in \mathbb{N}$ such that $\mu\left(P_{m}^{\perp} P G_{n} P^{\perp} Q_{k}\right)<\varepsilon$ for each $m \geq n_{k}$. For $\varepsilon=\frac{1}{2 k}\left(1-\sum_{j=1}^{k} \alpha_{j}\right)$ we select $n_{k}$ such that $\mu\left(P_{m}^{\perp} P G_{j} P^{\perp} Q_{k}\right)<\frac{1}{2 k}\left(1-\sum_{j=1}^{k} \alpha_{j}\right)$ for each $j \leq k$ and $m \geq n_{k}$. We can select the subsequence $\left\{P_{n_{k}}\right\}$ to be strictly increasing. Since $P_{n_{k+1}}-P_{n_{k}} \succeq P^{\perp} \succeq Q_{k}-Q_{k+1}$, there exists a partial isometry $V_{k}$ with initial projection $Q_{k}-Q_{k+1}$ and final projection $F_{k}<P_{n_{k+1}}-P_{n_{k}}$. So we have a sequence of partial isometries $\left\{V_{k}\right\}$ with initial projections and final projections mutually orthogonal. We define $T_{0}=\sum_{k=1}^{\infty} V_{k}$. The operator $T_{0}$ is a partial isometry and $T_{0} \in P \mathscr{M} P^{\perp}$. It is clear that $P_{n_{k}}^{\perp} T_{0} Q_{k}=P_{n_{k}}^{\perp} T_{0}$ is also a partial isometry with $\mu\left(P_{n_{k}}^{\perp} T_{0} Q_{k}\right)=1-\sum_{j=1}^{k-1} \alpha_{j}$ for $k \geq 2$.

Claim. $d\left(T_{0}, \mathscr{I}_{2}\right) \geq 1$.

Proof of the claim. Let $n_{0}$ be an arbitrary number in $\mathbb{N}$. Let $A_{j}, B_{j} \in$ $\operatorname{alg} \beta$ and $T_{j} \in P \mathscr{G} P^{\perp}$ for $j=1,2, \ldots, n_{0}$. Then the operator in the form of $\sum_{j=1}^{n_{0}} A_{j} T_{j} B_{j}$ is norm-dense in $\mathscr{J}_{2}$. Take an arbitrary such operator $\sum_{j=1}^{n_{0}} A_{j} T_{j} B_{j}$. Since $T_{j} \in P \mathscr{G} P^{\perp}=\left\{P G_{k} P^{\perp}\right\}$, there is a $k(j) \in \mathbb{N}$ such that $T_{j}=P G_{k(j)} P^{\perp}$. Let $k_{0}=\max \left\{\max \left[k(j): j=1,2, \ldots, n_{0}\right], n_{0}\right\}$. Then $\mu\left(P_{k_{0}}^{\perp} T_{j}\right)<\frac{1}{2 k_{0}}\left(1-\sum_{j=1}^{k_{0}} \alpha_{j}\right), \quad 1 \leq j \leq k_{0}$.

By Lemma 5 we have

$$
\begin{gathered}
\mu\left(P_{k_{0}}^{\perp}\left(\sum_{j=1}^{n_{0}} A_{j} T_{j} B_{j}\right) Q_{k_{0}}\right) \leq \sum_{j=1}^{n_{0}} \mu\left(P_{k_{0}}^{\perp} A_{j} T_{j} B_{j} Q_{k_{0}}\right) \leq \sum_{j=1}^{n_{0}} \mu\left(P_{k_{0}}^{\perp} T_{j}\right) \\
\leq n_{0} \frac{1}{2 k_{0}}\left(1-\sum_{j=1}^{k_{0}} \alpha_{j}\right) \leq \frac{1}{2}\left(1-\sum_{j=1}^{k_{0}} \alpha_{j}\right)<1-\sum_{j=1}^{k_{0}-1} \alpha_{j} \\
=\mu\left(P_{k_{0}}^{\perp} T_{0}\right)=\mu\left(P_{k_{0}}^{\perp} T_{0} Q_{k_{0}}\right) .
\end{gathered}
$$

By Lemma 5 again,

$$
\left\|P_{k_{0}}^{\perp}\left(T_{0}-\sum_{j=1}^{n_{0}} A_{j} T_{j} B_{j}\right) Q_{k_{0}}\right\| \geq 1 .
$$

So, $\left\|T_{0}-\sum_{j=1}^{n_{0}} A_{j} T_{j} B_{j}\right\| \geq 1$. That is, $d\left(T_{0}, \mathscr{I}_{2}\right) \geq 1$.

Next we prove that $T_{0} \notin[(\operatorname{alg} \beta) \mathscr{G}(\operatorname{alg} \beta)]$. It suffices to prove that $\lim \left\|P_{k_{0}}^{\perp} R Q_{k_{0}}\right\|=0$ for each element in $\mathscr{I}_{1}$ or in $\mathscr{I}_{3}$. This is clear by the Ringorose Criterion. 
Case 2. There is a projection $Q^{\prime} \in \beta, Q^{\prime}>P$, and $Q^{\prime}-P$ is a finite atom. In this case we can simply assume that $P^{\perp}$ is the finite atom. By the Ringrose Criterion (Theorem 2), we have $P^{\perp} \mathscr{G} P^{\perp}=\{0\}$. Since the factor is of type $\mathrm{II}_{\infty}$, we can refine the nest into a new nest $\beta^{\prime}$ by inserting a strictly decreasing sequence of projection $\left\{Q_{n}\right\}$ with limit point $P$ into the nest $\beta$. The nest $\beta^{\prime}$ is in Case 1. Using the same construction (with $\beta^{\prime}$ ) as in the proof of Case 1, we prove that there exists an operator $T_{0} \in P \mathscr{I}_{c} P^{\perp}$ such that

$$
\left\|P_{k_{0}}^{\perp}\left(T_{0}-\sum_{j=1}^{n_{0}} A_{j} T_{j} B_{j}\right)\right\| \geq 1 .
$$

So $d\left(T_{0}, \mathscr{I}_{2}\right) \geq 1$. Since $\mathscr{I}_{3}=\{0\}$ and $\lim \left\|P_{k_{0}}^{\perp} R\right\|=0$ for each element in $\mathscr{I}_{1}$ by the Ringorose Criterion again, we have proven Case 2. Theorem 4 is proven.

\section{REFERENCES}

1. X. Dai, Norm-principal bimodules of nest algebras, J. Funct. Anal. 90 (1990), 369-390.

2. __ Norm-principal bimodules of nest algebras II, preprint.

3. K. R. Davidson, Nest algebras, Pitman Res. Notes in Math. Ser., vol. 191, Longman Sci. Tech., Harlow, 1988.

4. R. V. Kadison and J. R. Ringrose, Fundamentals of the theory of operator algebras, vols. I and II, Academic Press, New York, 1983, 1986.

5. Frank Gilfeather and David Larson, Nest-subalgebras of von Neumann algebras. Adv. in Math. 46 (1982), 171-199.

6. J. L. Orr, On generators of the radical of a nest algebra, J. London Math. Soc. (2) 40 (1989), 547-562.

7. J. R. Ringrose, On some algebras of operators, Proc. London Math. Soc. (3) 15 (1965), 61-83.

Department of Mathematics, University of North Carolina, Charlotte, North CarOLINA 28223

E-mail address: xdai@unccsun.uncc.edu 